\title{
Intracorporeal esophagojejunal anastomosis after laparoscopic total gastrectomy for patients with gastric cancer.
}

\section{$\operatorname{AUTHOR}(\mathrm{S})$ :}

Okabe, Hiroshi; Obama, Kazutaka; Tanaka, Eiji; Nomura, Akinari; Kawamura, Jun-ichiro; Nagayama, Satoshi; Itami, Atsushi; Watanabe, Go; Kanaya, Seiichiro; Sakai, Yoshiharu

\section{CITATION:}

Okabe, Hiroshi ... [et al]. Intracorporeal esophagojejunal anastomosis after laparoscopic total gastrectomy for patients with gastric cancer.. Surgical endoscopy 2009, 23(9): 2167 2171

\section{ISSUE DATE:}

2009-09

URL:

http://hdl.handle.net/2433/93003

\section{RIGHT:}

c Springer Science+Business Media, LLC 2008; This is not the published version. Please cite only the published version.; この論文は出版社版で ありません。引用の際には出版社版をご確認ご利用ください。 


\section{Full Title}

Intracorporeal esophago-jejunal anastomosis after laparoscopic total gastrectomy for patients with gastric cancer

\section{Authors}

Hiroshi Okabe, $M D, \mathrm{PhD}^{1)}$, Kazutaka Obama, $\mathrm{MD}, \mathrm{PhD}^{1)}$, Eiji Tanaka $\mathrm{MD}, \mathrm{PhD}^{1)}$, Jun-ichiro Kawamura MD, $\mathrm{PhD}^{1)}$, Akinari Nomura $\mathrm{MD}^{1)}$, Satoshi Nagayama, MD, $\mathrm{PhD}^{1)}$, Atsushi Itami, MD, $\mathrm{PhD}^{1)}$, Go Watanabe, $\mathrm{MD}, \mathrm{PhD}^{1)}$, Seiichiro Kanaya, MD, $\mathrm{PhD}^{2)}$, Yoshiharu Sakai, MD, $\mathrm{PhD}^{1)}$

\section{Affiliation}

1) Department of Surgery,

Kyoto University Graduate School of Medicine

54 Kawahara-cho, Shogoin, Sakyo-ku, Kyoto, 606-8507, Japan

2) Department of Surgery,

Fujita Health University, School of Medicine

1-98 Dengakugakubo, Kutsukake-cho, Toyoake, Aichi 470-1192, Japan.

\section{Corresponding Author}

Hiroshi OKABE

Department of Surgery, Kyoto University Graduate School of Medicine

54 Kawahara-cho, Shogoin, Sakyo-ku, Kyoto, 606-8507, Japan

e-mail:hokabe@kuhp.kyoto-u.ac.jp

phone:+81-75-751-3227, fax:+81-75-751-4390 


\section{Abstract}

To facilitate acceptance of laparoscopic total gastrectomy (LTG) for patients with upper gastric cancer, a simple and secure technique of reconstruction is necessary. We made some modifications to the extracorporeal reconstruction method using linear staplers and developed a new method of intracorporeal esophagojejunal anastomosis, which does not require hand sewing. First, we transected the esophagus under laparoscopy. This allowed us to perform the appropriate pathological evaluation of the proximal margin. Second, to aid in the safe insertion of an endoscopic linear stapler into the lumen of the esophagus and firing with the edge of the jejunal limb, we used a naso-gastric tube as an insertion guide. Third, we roughly closed the entry hole with hernia staplers before the final closure. This allowed us to apply an endoscopic linear stapler very close to the opening edge to leave a wide anastomotic lumen, and at the same time to maintain a safety margin for complete closure. Using this anastomotic procedure, we successfully performed total laparoscopic esophagojejunal anastomosis in 16 patients without any postoperative anastomotic complications. We believe our procedure is a secure and reliable method for reconstruction after LTG and will facilitate adoption of LTG as a surgical option for patients with upper gastric cancer.

\section{Key Words}

total gastrectomy, laparoscopic surgery, esophagojejunostomy, gastric cancer 


\section{Introduction}

A simple and secure technique of reconstruction is necessary to facilitate acceptance of laparoscopic total gastrectomy (LTG) for patients with upper gastric cancer. We previously reported the procedure of extracorporeal Roux-en $Y$ reconstruction, which can be completed by linear staplers via a minilaparotomy[1]. We have recently modified the procedure and developed a new intracorporeal method of linear-stapled esophagojejunal anastomosis.

\section{Patients and Methods}

\section{Patients}

Between September 2006 and January 2008, 16 patients (11 males and 5 females) with gastric cancer underwent LTG at our institution. These patients had a median age of 70 years (range: 39 to 81 years) and a body mass index of 20.8 (range: 16.7 to 27.6 ). All patients were diagnosed with gastric cancer, which was located in the upper third of the stomach without lymph node involvement. These diagnoses were based on preoperative examinations, including gastrointestinal endoscopy, upper gastrointestinal series, and abdominal CT scan.

\section{Surgical technique}

The patient was placed in a modified lithotomy position. The endoscope was inserted via the umbilical port and total gastrectomy was performed using four operating ports, as previously described (Fig 1) [1]. After the abdominal esophagus was completely exposed by division of the anterior and posterior vagal trunks, gastrectomy was completed by transection of the esophagus. To make the subsequent anastomosis easier, the esophagus was transected using ETS-FLEX 45 (Ethicon Endo-surgery), while it was rotated by about 45 degrees counter clockwise (Fig 2A).

The umbilical trocar wound was extended and the stomach was exteriorized. The proximal margin of the specimen was pathologically examined when necessary. A jejunal loop about $20 \mathrm{~cm}$ distal to the ligament of Treitz was marked with a dye and pulled out through the wound and transected, and then side-to-side jejunojejunostomy (Y-anastomosis) was performed using ETS-FLEX 45 to create a 50-cm Roux-en-Y limb. After a small hole was made at the end of the Roux-en-Y limb on the anti-mesenteric side, the $Y$-anastomosis was placed back into the abdominal cavity and pneumoperitoneum was re-established.

A small hole was made on the left-dorsal side of the esophageal stump. For easier detection of the lumen of the esophagus, a naso-gastric tube was advanced through the hole (Fig 3A). The Roux-en-Y limb was then brought up via the antecolic route to create an esophagojejunostomy. Both cut ends of the esophagus and the jejunal limb were placed side by side with their blind ends beside each other. ETS-FLEX 45 was applied through the left lower trocar to create a single 
lumen between the left dorsal side of the esophagus and the jejunal limb (Fig 2B and 3B). It is important to avoid insertion of the stapler into a 'pseudo' lumen, which is made artificially between the submucosal and the muscular layers. To assure that the staple jaw was inserted into the 'true' lumen of the esophagus, we inserted the stapler following the naso-gastric tube while it was slowly retracted. After the entry hole was roughly closed with ENDO UNIVERSAL ${ }^{\mathrm{TM}} 65^{\circ}$ staplers (Tyco Healthcare), which are generally used for fixation of mesh in hernia repair, ETS-FLEX 45 was applied again through the right lower trocar so that all hernia staplers were removed and the closure was completed (Fig 2C, 2D, 4A-4C). In some cases, further application was necessary to complete the closure.

An air leakage test was performed to confirm the tightness of the anastomosis; a flat type suction drain was inserted from the upper right port and placed near the esophago-jejunal anastomosis to the left subphrenic space (Fig 4D). The operation was completed by closure of all trocar wounds.

\section{Results}

The mean operative time for 16 patients was $325 \pm 68 \mathrm{~min}$, and the estimated blood loss was $195 \pm 197 \mathrm{~g}$. All operations were curative and the mean number of retrieved lymph nodes was 47 \pm 13 . In one patient, the ninth patient among the series, intracorporeal anastomosis failed because a naso-gastric tube was caught between the jaws of an endostapler. He required thoracotomy to redo the anastomosis. In the remaining 15 patients, Roux-en-Y reconstructions were successfully performed using the intracorporeal method.

One patient developed intraabdominal abscess and one patient had aspiration pneumonia, both of which resolved following treatment with antibiotics. We did not experience stenosis or leakage of the anastomosis. Postoperative endoscopy demonstrated large and nearly 'circular' anastomosis (Fig 5). Fluorography demonstrated smooth passage of contrast media through the anastomosis, which resembled the shape after an end-to-end anastomosis (Fig 6). The median day to the resumption of intake of clear liquids was the third postoperative day and ingestion of food occurred on the following day. Except for the two patients with postoperative complications, drip infusion was discontinued by the sixth postoperative day. The median postoperative hospital stay was 11 days. There was no mortality.

\section{Discussion}

With improvements of endoscopic surgical devices and the surgeons' skills, laparoscopic procedures for adequate lymph node dissection for gastric cancer patients are becoming established[2,3]. However, laparoscopic reconstruction, especially esophago-jejunal anastomosis after LTG, is still technically demanding. In most reports of LTG, circular staplers are 
used to make an esophago-jejunostomy[4,5]. Since there is no circular stapler specially designed for endoscopic surgery, and it is difficult to operate circular staplers under the limited laparoscopic view, surgeons preferred extracorporeal use of circular staplers. To perform intracorporeal anastomosis, some groups have reported linear stapled esophago-jejunostomy [6,7]. In these reports, to prevent possible stenosis, the access opening is closed with hand sutures, which takes longer to perform and requires a higher degree of surgical skill. We previously reported an easier procedure of extracorporeal linear stapled esophago-jejunostomy[1]. In this procedure, we anastomosed the jejunal limb to the left side of the esophagus, not to the conventionally used right side, allowing easy application of a linear stapler by using sufficient free working space. The access opening was then closed with linear staplers, assisted by lifting supporting sutures of the entry hole from the mini-laparotomy. While we believe our extracorporeal method is widely acceptable as a standard reconstruction after LTG because of its safety and simplicity, there were some possible disadvantages. First, although we successfully performed Roux-en-Y reconstruction through a $5-\mathrm{cm}$ mini-laparotomy in a series of patients, this would be more difficult in obese patients and extension of the laparotomy may be necessary. Second, because we created the esophagojejunal anastomosis before transection of the esophagus, precise evaluation of the oral margin of the esophagus was difficult. Thus, we did not apply the procedure for patients with tumors invading the esophagus.

In this paper, we reported a new method of intracorporeal linear stapled anastomosis. Since the whole procedure is completed under laparoscopy, the method could be easily applied to obese patients. In the new method, to solve the potential problem of proximal margin, we transected the esophagus under laparoscopy before starting esophagojejunal anastomosis. This allows us to perform the appropriate pathological evaluation of the proximal margin. We also made other modifications to accomplish intraabdominal anastomosis securely and easily without handsewing. First, in contrast to the previously reported linear stapled esophagojejunostomy, we anastomosed the jejunal limb to the left side of the esophagus, not to the conventionally used right side, allowing easy application of a linear stapler by using sufficient free working space in the left subphrenic area. As a result of the rotated transection of the esophagus, the jejunum was anastomosed to the left-dorsal edge of the esophagus. This rotation is important to make the subsequent closure through the right lower trocar easier. Second, to assist in the safe insertion of an endoscopic linear stapler into the true lumen of the esophagus, we used a naso-gastric tube as an insertion guide. Since the esophagus is often shortened and withdrawn into the mediastinum after transaction, identification of the lumen is sometimes difficult. Pushing the naso-gastric tube through the hole on the esophageal stump is quite helpful to identify the lumen. However, we experienced a case, in which a tube was involved in the anastomosis. Therefore, it is recommended to confirm that the tube is not caught between the jaws prior to closing the anvil. 
Third, to complete anastomosis with endoscopic linear staplers without increased risk of leakage or stenosis, we roughly closed the entry hole with hernia staplers before final closure. This allowed us to apply an endoscopic linear stapler very close to the opening edge to leave a wide anastomotic lumen, and at the same time to maintain the safety margin for complete closure. Although we prefer to use a linear stapler to close the entry hole, it would be difficult in the case in which the esophagus was transected at a high level to keep a proximal margin. We closed the entry hole by hand suturing in such a case.

With all of these modifications, we believe our new procedure could become one of the standard methods for reconstruction after LTG, and facilitate the acceptance of LTG as a surgical option for patients with upper gastric cancer. 


\section{Figure Legends}

Fig. 1.

Placement of surgical ports. 12-mm ports were used for ports A, C, D, and E. A 5-mm port was used for B. A flexible endoscope was introduced via port A. A liver retractor was introduced from F. Fig. 2.

Schematic outline of intracorporeal linear-stapled esophagojejunal anastomosis. A. Esophagus is transected while being rotated 45-degree counter clockwise. B. The left dorsal side of the esophagus and the jejunal limb are anastomosed by an endoscopic linear stapler. C. The entry hole is roughly closed with staplers for tissue approximation. D. Closure is completed by application of an endoscopic linear stapler.

Fig. 3.

Generation of the common lumen between the esophagus and the jejunal limb. A. An N-G tube is pushed through the hole to identify the lumen for proper insertion of an endoscopic linear stapler.

B. An endoscopic linear stapler is applied between the left dorsal side of the esophagus and the jejunal limb. N-G tube: Naso-gastric tube, E: Esophagus, J: Jejunal limb

Fig. 4.

Final closure of the entry hole by an endoscopic linear stapler. A, B. The common hole is roughly closed with staplers for tissue approximation (ENDO UNIVERSAL ${ }^{\mathrm{TM}}$ 65). C. Endoscopic linear stapler is applied to complete closure. D. Laparoscopic view of the esophagojejunostomy. E: Esophagus, J: Jejunal limb.

Fig. 5.

A. Endoscopic view of the anastomosis at six months after operation. B. Solid line shows the anastomotic line generated by the first application of the stapler, and the dotted line indicates the closure line by the second application of the stapler.

Fig. 6.

Fluorography on the seventh postoperative day demonstrates smooth passage of oral hydrosoluble contrast without leakage or stenosis of the anastomosis. 


\section{References}

1 Okabe $H$, Satoh $S$, Inoue $H$, Kondo $M$, Kawamura J, Nomura A, Nagayama $S$, Hasegawa S, Itami A, Watanabe G, Sakai Y: Esophagojejunostomy through minilaparotomy after laparoscopic total gastrectomy. Gastric Cancer 2007;10:176-180.

2 Fujiwara M, Kodera Y, Misawa K, Kinoshita M, Kinoshita T, Miura S, Ohashi N, Nakayama G, Koike M, Nakao A: Longterm outcomes of early-stage gastric carcinoma patients treated with laparoscopy-assisted surgery. J Am Coll Surg 2008;206:138-143.

3 Huscher C, Mingoli A, Sgarzini G, Brachini G, Binda B, Di Paola M, Ponzano C: Totally laparoscopic total and subtotal gastrectomy with extended lymph node dissection for early and advanced gastric cancer: Early and long-term results of a 100-patient series. Am J Surg 2007;194:839-844; discussion 844.

4 Dulucq J, Wintringer P, Perissat J, Mahajna A: Completely laparoscopic total and partial gastrectomy for benign and malignant diseases: A single institute's prospective analysis. J Am Coll Surg 2005;200:191-197.

5 Usui S, Yoshida T, Ito K, Hiranuma S, Kudo S, Iwai T: Laparoscopy-assisted total gastrectomy for early gastric cancer: Comparison with conventional open total gastrectomy. Surg Laparosc Endosc Percutan Tech 2005;15:309-314.

6 Huscher C, Mingoli A, Sgarzini G, Sansonetti A, Lirici M, Napolitano C, Piro F: Videolaparoscopic total and subtotal gastrectomy with extended lymph node dissection for gastric cancer. Am J Surg 2004;188:728-735.

7 Tanimura S, Higashino M, Fukunaga $\mathrm{Y}$, Kishida S, Ogata A, Fujiwara $\mathrm{Y}$, Osugi H: Laparoscopic gastrectomy with regional lymph node dissection for upper gastric cancer. $\mathrm{Br} \mathrm{J}$ Surg 2007;94:204-207. 


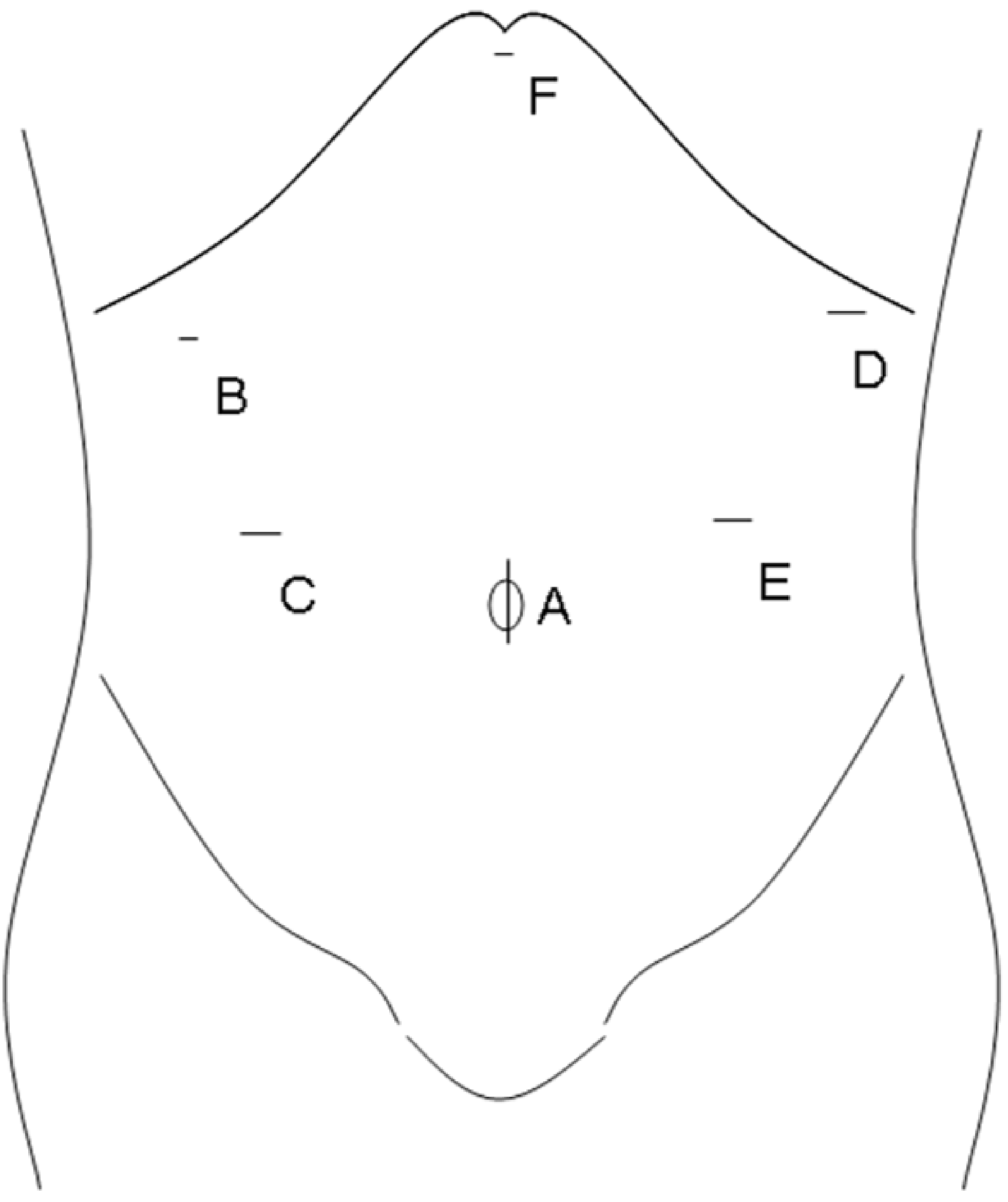




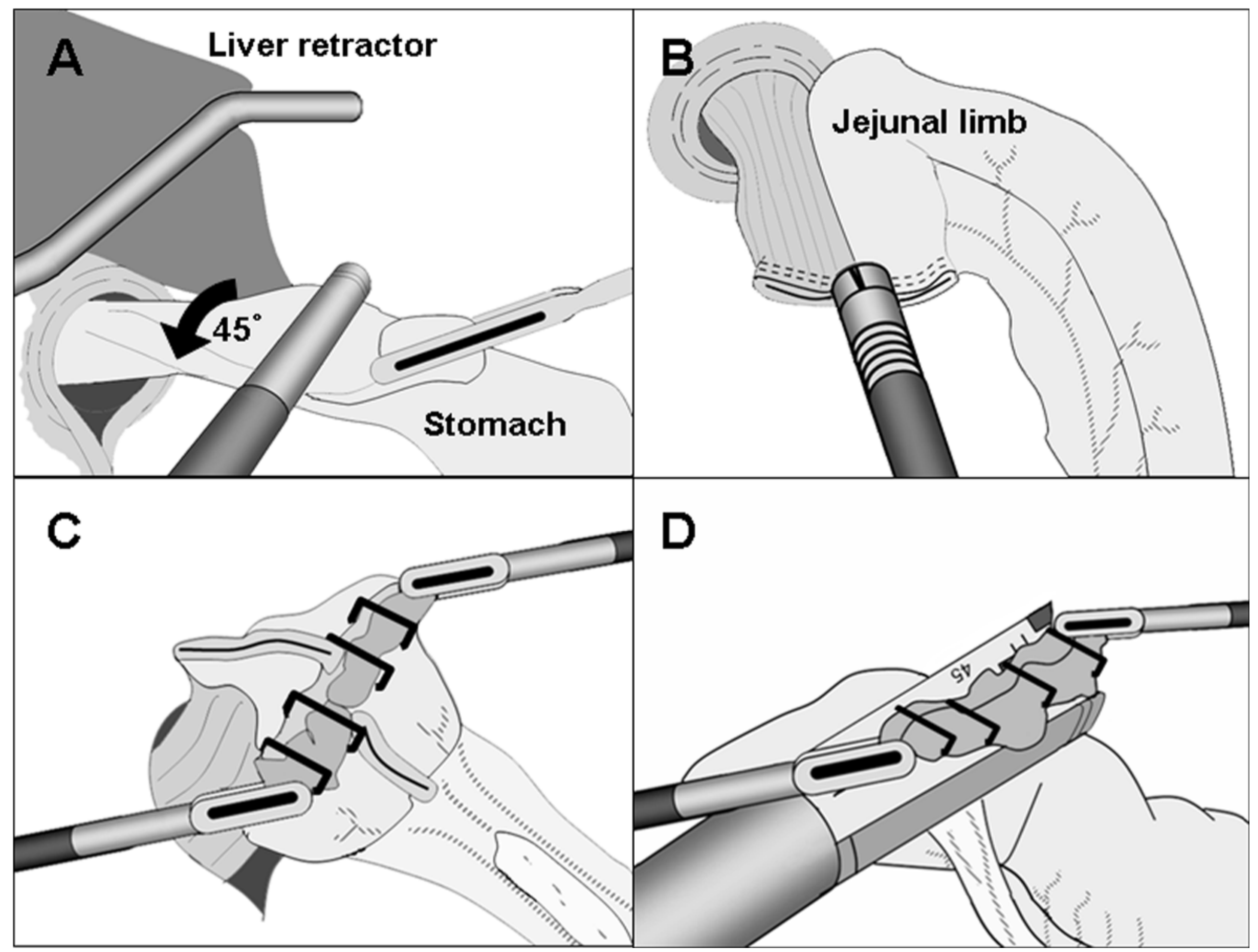




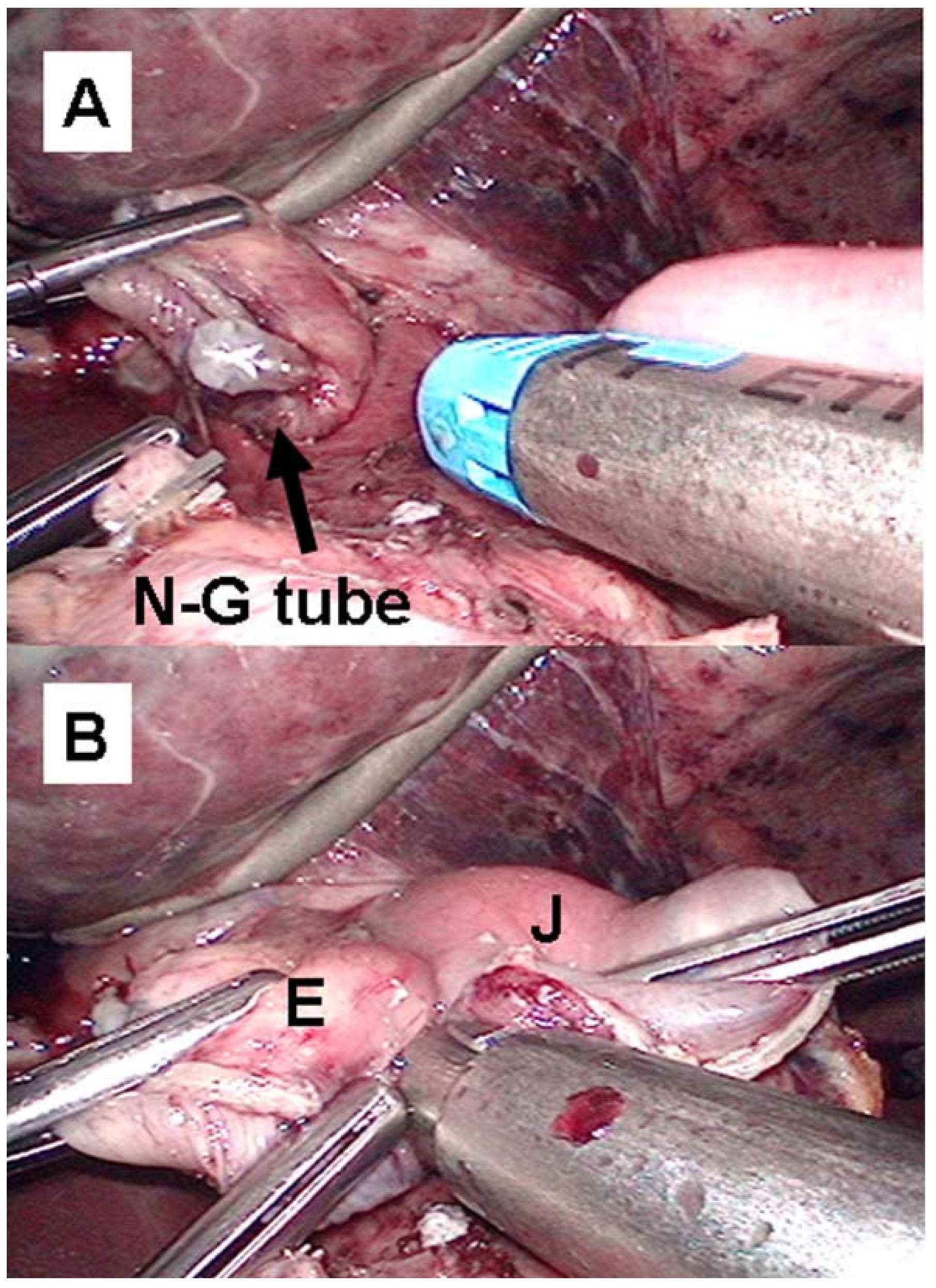




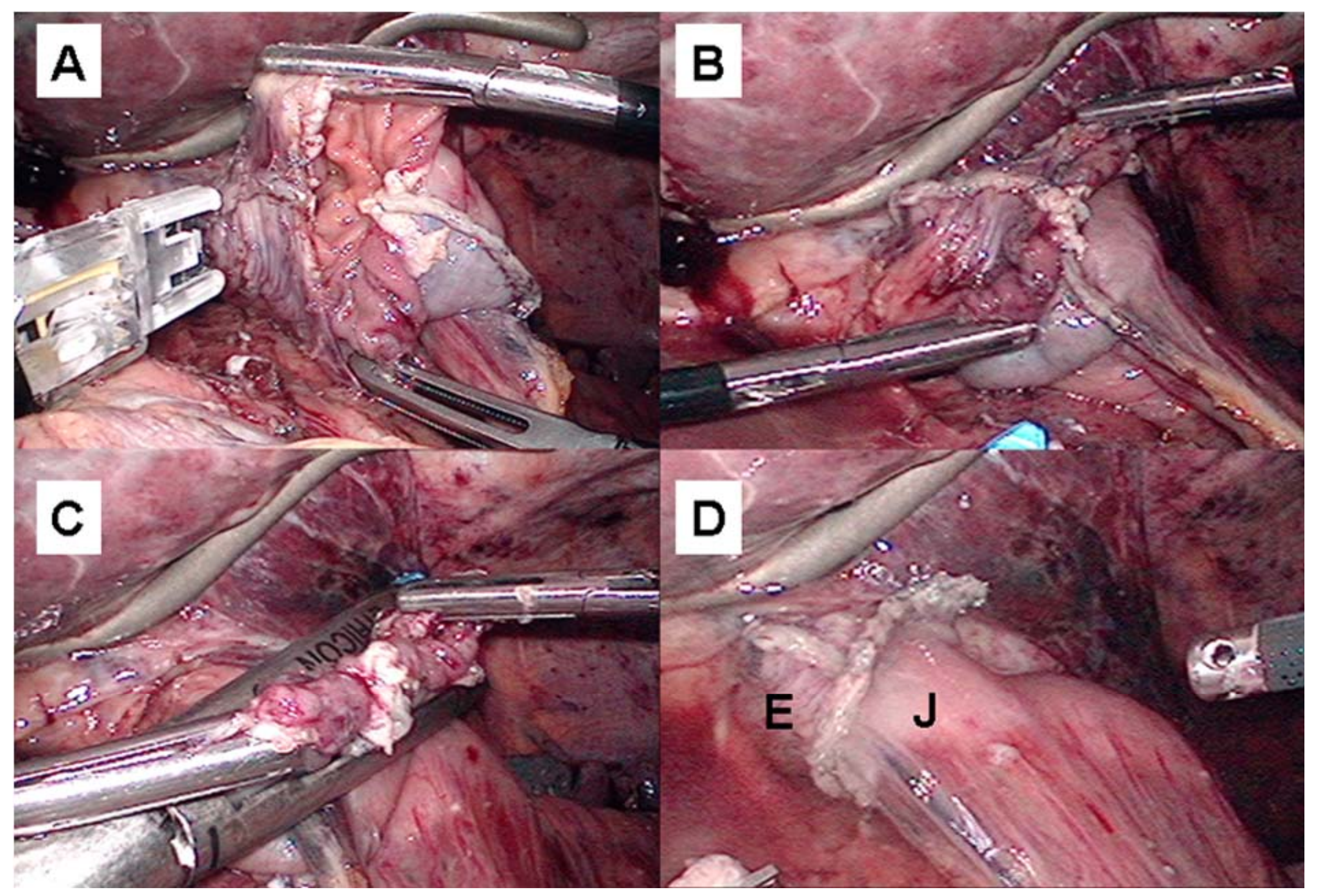




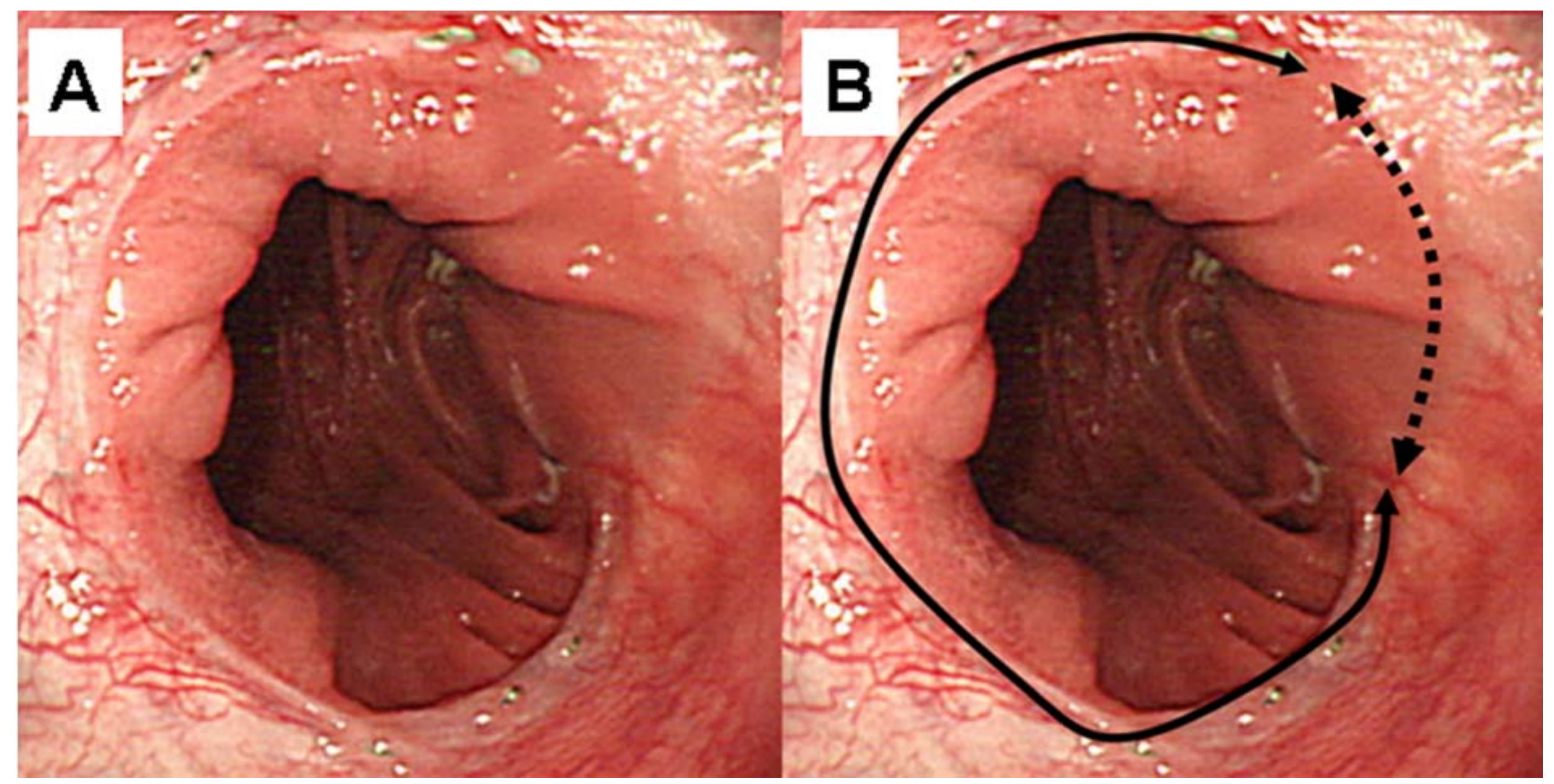




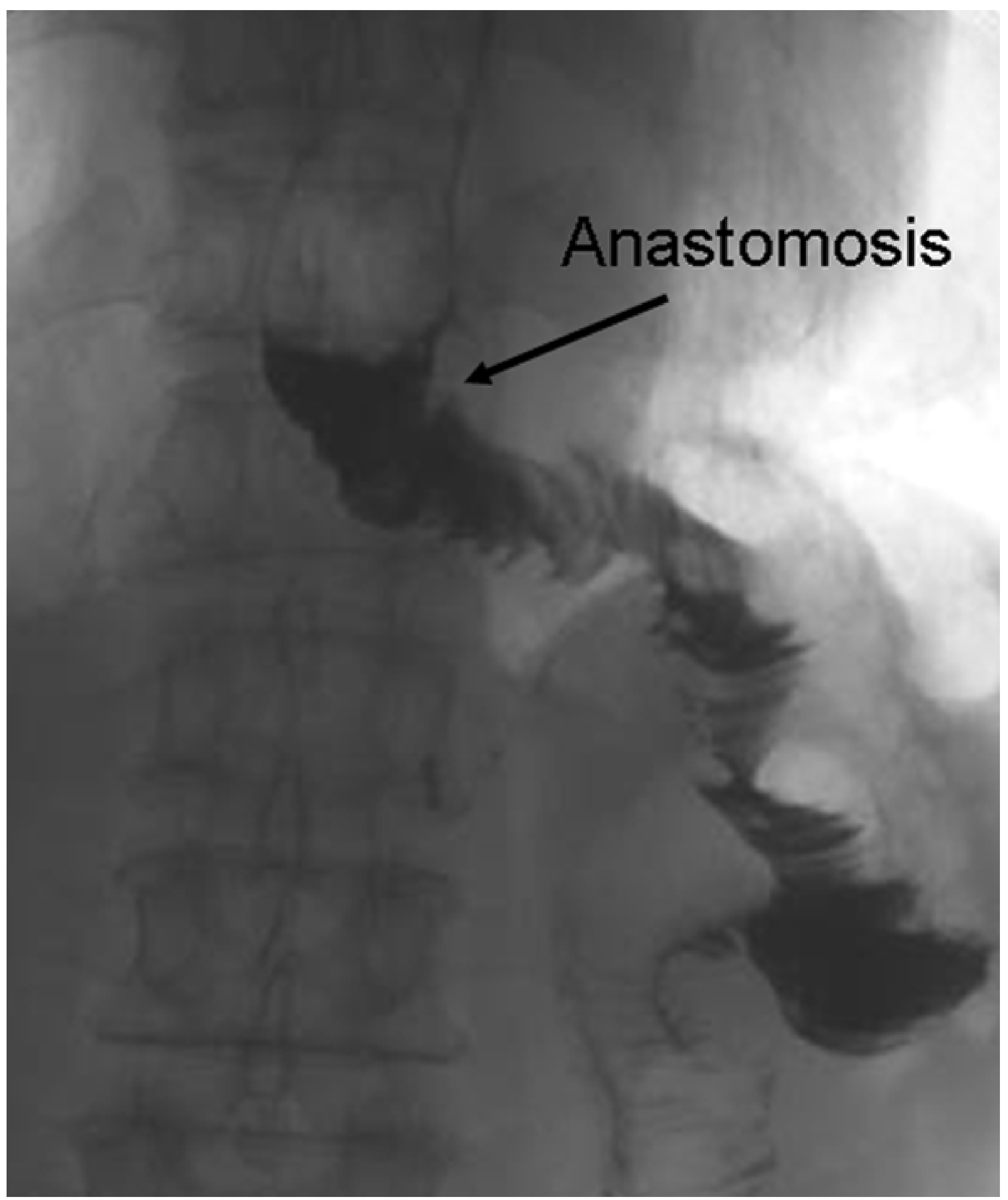

